\title{
Decidual Vasculopathy in Preeclampsia and Spiral Artery Remodeling Revisited: Shallow Invasion versus Failure of Involution
}

\author{
Peilin Zhang, MD, PhD ${ }^{1}$ \\ ${ }^{1}$ Department of Pathology, NYP Brooklyn Methodist Hospital, \\ Brooklyn, New York \\ Am J Perinatol Rep 2018;8:e241-e246.
}

\begin{abstract}
Address for correspondence Peilin Zhang, MD, PhD, Department of Pathology, NYP Brooklyn Methodist Hospital, 506 Sixth Street, Brooklyn, NY 11215 (e-mail: Pez9008@nyp.org).
\end{abstract}

\begin{abstract}
Decidual vasculopathy is commonly associated with preeclampsia and develops in the late pregnancy in the uterine spiral arteries, which were previously remodeled by the extravillous trophoblasts. In normal early pregnancy, trophoblasts invade into the spiral artery, leading to vascular transformation, and this transformation is found to be associated with phenotypic switch of the endovascular trophoblasts to express CD56, a maternal protein likely from the natural killer (NK) cells. These endovascular trophoblasts are diminished at term. The decidual vessels are also returned to near normal at the delivery. Both the uterus and the uterine spiral arteries undergo involution after

\section{Keywords}

- decidual vasculopathy

- preeclampsia

- CD56

- natural killer

- spiral artery remodeling

- involution delivery. In preeclampsia, the endovascular trophoblasts are present within the vessel wall associated with the persistence of switched phenotype similar to those seen in the early implantation. The persistence of the endovascular trophoblasts in decidual vasculopathy indicates a failure to return to normal vessels in preeclampsia, thus suggesting a potential mechanism of pathogenesis. NK cells seem critical not only for early implantation and spiral artery remodeling but also for the development of decidual vasculopathy in preeclampsia. In this short review, some critical aspects of decidual vasculopathy in normal pregnancy and preeclampsia are reexamined and a new hypothesis is proposed.
\end{abstract}

\section{Preeclampsia}

Preeclampsia is a heterogeneous group of clinical syndromes affecting 5 to $8 \%$ pregnancies. Preeclampsia can be classified on the basis of the severity of clinical symptoms as mild, moderate, or severe, or gestational age as early-onset or late-onset. ${ }^{1-3}$ The early-onset preeclampsia (before 34 weeks) is considered a different disease entity due to the high probability of adverse maternal and fetal complications. Early-onset preeclampsia is thought to be mediated by the placenta with defective trophoblast invasion of the maternal vessels, and defective angiogenesis from the placental development. The maternal serum angiogenic factor, placental growth factor (PIGF), and Doppler ultrasound examination of the uterine artery in the second

received

June 27, 2018

accepted after revision

September 10, 2018
DOI https://doi.org/

10.1055/s-0038-1675348. ISSN 2157-6998. trimester were shown to be useful as predictive tools. ${ }^{4}$ Placental pathology commonly associated with preeclampsia is centered on the ischemic changes such as villous tissue infarcts, maternal vascular malperfusion, and vascular thrombosis. The late-onset preeclampsia, however, appears to be associated with less adverse maternal-fetal complications and different hemodynamics of the maternal circulation. ${ }^{2}$ Serum PIGF and ultrasound imaging of the uterine artery seem less useful for late-onset preeclampsia. Placental examination commonly fails to show significant histopathological changes within the villous tissue or vasculatures. A large body of literature depicting preeclampsia and its defective trophoblast invasion and maternal vascular malperfusion appear fit better
Copyright $\odot 2018$ by Thieme Medical Publishers, Inc., 333 Seventh Avenue, New York, NY 10001, USA. Tel: +1(212) 584-4662.
License terms

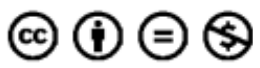


to the early-onset preeclampsia, but early-onset preeclampsia represents approximately 10 to $20 \%$ of overall preeclampsia patients, ${ }^{1}$ and the remaining majority of preeclampsia patients is a mystery in their disease mechanism and pathogenesis.

\section{Decidual Vasculopathy, Preeclampsia, and Implantation}

Decidual vasculopathy is commonly associated with maternal preeclampsia and hypertension. Decidual vasculopathy is characterized by the vascular transformation within the decidua. There are three morphological types of decidual vasculopathy, namely acute atherosis, fibrinoid medial necrosis, and mural arterial hypertrophy. ${ }^{5,6}$ These morphological features could represent the various stages of the same pathological process. Decidual vasculopathy was first described in detail in 1945 by Arthur Hertig as "acute degenerative arteriolitis," and there were three phases of the morphological changes. ${ }^{7}$ The first phase was described as "collection of foamy fat-laden mononuclear leukocytes or phagocytes" beneath the intima of the spiral artery followed by the "fibrinoid degeneration" of the media, which is superseded by "fibroblastic proliferation" of intima and results in near-complete "obliteration" of the lumen. These vascular lesions were also studied in details by others including Marais in 1962. ${ }^{8,9}$ These seminal changes of the decidual vessels in vasculopathy and their descriptive characteristics remain essentially unchanged in the modern textbooks of placental pathology today. Acute atherosis is also named in similarity to adult atherosclerosis in which foamy macrophages with lipid/cholesterol are deposited within the wall or the lumen of the vessel. Acute atherosis in preeclampsia shows several foamy cells within the vascular wall or lumen, and these foamy cells are believed to be "macrophages" phenotypically reactive to CD68 by immunostaining with similar characteristics to adult atherosclerosis. ${ }^{10}$ The morphological features are named "fibrinoid medial necrosis" when the vascular wall consists of mainly eosinophilic fibrinlike material free of cellular components, and there are mixed immune deposits including immunoglobulin (Ig) G, IgM, and complement C3 within the eosinophilic material by immunostaining or immune-fluorescent detection methods. ${ }^{11}$

The replacement of vascular wall including the muscular media, intima, and adventitia with fibrinlike eosinophilic material and disappearance of endothelial cells in late pregnancy associated with preeclampsia is morphologically similar to those seen in spiral artery remodeling in early implantation. Implantation and spiral artery remodeling were performed by the fetal extravillous trophoblasts. ${ }^{5,12,13}$ In early implantation, there are extravillous trophoblasts invading into the lumen of the vessels, ranging from a few to a large number with aggregates forming a plug in the vascular lumen, depending upon the gestational age of the placenta. ${ }^{12,14}$ Trophoblastic invasion into the vascular lumen represents the first step of establishing the intervillous circulation, and the entire intervillous space is subsequently lined by the syncytiotrophoblasts of the villous tree without endothelial cells or smooth muscle. The spiral artery remodeling not only is a physiological process to allow dramatic maternal blood volume expansion and fetal development but also seems critical for the maintenance of maternal blood pressure in the presence of increasing volume expansion (discussed later). I have first noted that the CD68-reactive "macrophages" in acute atherosis in preeclampsia are immunoreactive to pan-cytokeratin (AE1/AE3), in addition to CD68, and these cells are phenotypically consistent with fetal trophoblasts. ${ }^{15}$ Unlike other trophoblasts within the villous tissue or decidua (interstitial), these intravascular (endovascular) trophoblasts express CD56, a defining marker of natural killer (NK) cells of likely maternal origin. ${ }^{15}$ These vascular changes in late gestation in preeclampsia are morphologically similar, if not identical, to those in early implantation sites where decidual spiral artery remodeling occurs with endovascular trophoblasts. The morphological spectrum of the decidual vasculopathy in preeclampsia can be unified as "CD56-related vasculopathy" to imply the potential pathogenic role of the persistence of fetal trophoblasts within the maternal vessels. ${ }^{15}$ Maternal spiral artery remodeling is a prerequisite step for all successful pregnancy. ${ }^{16}$ Given the fact that the endovascular trophoblasts with phenotypic switch are required for maternal vascular remodeling, which occurs in all successful pregnancies, these endovascular trophoblasts must have undergone a cell death process such as apoptosis during pregnancy since no endovascular trophoblasts are present at the normal term placentas. To simplify, normal pregnancy starts with spiral artery remodeling by the trophoblasts and concludes with "return to normal or near-normal" maternal spiral artery. In contrast, the decidual vasculopathy in preeclampsia shows the persistence of various numbers of trophoblasts in the vessels similar to those in early implantation. It seems logical to propose that endovascular trophoblasts with phenotypic switch to express CD56 in early implantation is related to preeclampsia in late pregnancy, and persistence of these endovascular trophoblasts from early implantation to late gestation suggests failure of a process to "return to normal or near-normal," and this failure to "return to normal" is at least a part of the decidual vasculopathy in preeclampsia (-Fig.1).

Mural arterial hypertrophy is characterized by the presence of arteries of thickened medial wall surrounded by scattered lymphocytes (NK cell). ${ }^{17,18}$ It seems to represent a failure of trophoblast invasion into the vessels, leading to failure of spiral artery remodeling. It is commonly associated with maternal hypertension and could represent preeclampsia superimposed on preexisting chronic hypertension. This particular vascular change is difficult to study in both early implantation and late pregnancy due to the lack of specific markers similar to CD56 expression in trophoblasts. Lack of spiral artery remodeling can lead to pregnancy loss, and this may represent vascular changes in true hypertension since these morphological changes are similar to those in hypertensive vasculopathy in adult nonpregnant population. It is also known from epidemiological data that chronic hypertension poses two times higher risk of infertility. Whether mural hypertrophy is related to the failure of phenotypic switch of the trophoblasts leading to failure of trophoblastic remodeling of the spiral artery or the phenotypic switch of 


\section{Implantation EVT CD56+}

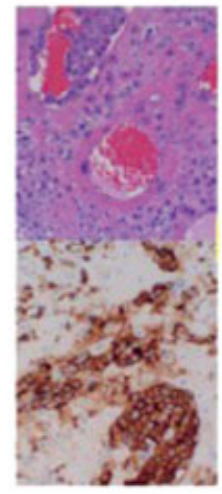

Physiological
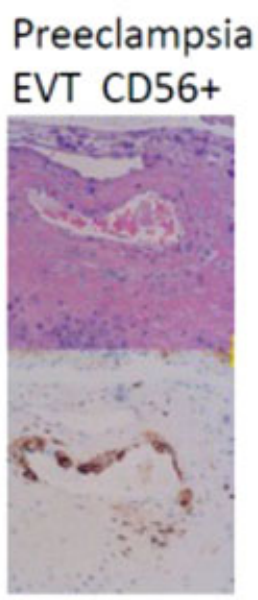

Pathological

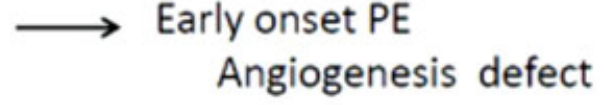

Small placenta

Infarcts

Thrombosis

IUGR

\section{$\rightarrow$ Late onset PE \\ Normal development}

\section{$\rightarrow$ Other complications without PE}

Fig. 1 Endovascular trophoblasts (EVT) with phenotypic switch in implantation and persistence decidual vasculopathy in preeclampsia (PE) and other complications.

trophoblasts and vascular invasion are two separate issues that remain unclear. In a mice model deficient of interferon gamma (IFN-G) or reduction of uterine NK cells, the decidual spiral artery showed increased thickness of the vascular wall with a narrow lumen, ${ }^{19,20}$ in addition to decidual necrosis in late gestation, and these vascular changes in the murine model share significant similarities to human mural arterial hypertrophy. It is possible that mural arterial hypertrophy in humans is related to abnormal signaling of IFN-G and NK cells in early or late gestations since mural arterial hypertrophy can be identified in both early implantation sites in abortion specimens and preeclamptic placentas associated with significant fetal and placental complications. ${ }^{18,21}$ In our experience, mural arterial hypertrophy is commonly seen in placentas from preeclampsia or chronic hypertension (earlyonset preeclampsia) but not common in placentas with other complications such as diabetes without preeclampsia or hypertension. Quantification of NK cell number in human placentas with this type of vasculopathy or quantification of maternal serum IFN-G in maternal hypertension may provide an insight into whether mural arterial hypertrophy in pregnancy complication is related to NK cell function.

\section{Hypertension and Preeclampsia}

Blood pressure in physiological conditions is maintained through volume, cardiac output, and peripheral resistance in a complex manner, and regulation of blood pressure involves neuroendocrine system and local vascular regulatory systems including vasoconstrictive and vasodilative systems. In pregnancy, the maternal volume is increased up to $40 \%$, and the cardiac output is consequently increased. ${ }^{2}$ The peripheral resistance is decreased to accommodate not only the fetal growth requirement of nutrients and but also the maintenance of normal maternal blood pressure. In gestational hypertension or preeclampsia, the blood volume and maternal cardiac output are mostly similar to or lower than that in normal pregnancy, and the peripheral resistance appears to be increased and the local vascular regulation is affected. There are many endocrine factors and local vasoconstrictors/vasodilators involving the systematic regulation of blood pressure. ${ }^{2}$ A large body of literature on shallow trophoblastic invasion and inadequate spiral artery remodeling in pregnancy demonstrates a quantitative insufficiency of decrease of the maternal peripheral resistance as a mechanism of pathogenesis of preeclampsia. Shallow trophoblast invasion and inadequate spiral artery remodeling result in small placenta development and hypoxic/ischemic changes in placenta, such as infarcts, vascular thrombosis, and villous hypoplasia. ${ }^{22}$ This is especially important for early-onset preeclampsia. However, a majority of the preeclampsia is late-onset, with normal to large placentas without identifiable histopathological features on placental examination. Our data suggest an alternative explanation in that if the spiral artery remodeling by trophoblasts in early pregnancy (first and second trimester) remains the same in normal pregnancy and preeclampsia, subsequent failure to return to normal vascular structure at term could be pathogenically important to preeclampsia, and decidual vasculopathy in this setting could be the only pathological feature on placental examination. This "failure to return to normal" vessels at term could represent a new pathogenic mechanism.

Preeclampsia is clinically defined after 20 weeks of gestational age, and the defining feature of preeclampsia is maternal hypertension. It is essential to have hypertension for clinical diagnosis of preeclampsia in the clinical guideline. Consequently, it is important to examine the relationship between maternal vasculopathy and maternal hypertension. Our previous study examined 175 preeclamptic placentas, and we found $72.6 \%$ (127) of the preeclamptic placentas with all three morphological types of decidual vasculopathy. Meanwhile, we have examined 137 nonpreeclamptic placentas with decidual vasculopathy associated with other pregnancy complications without preeclampsia or hypertension. ${ }^{15}$ Our study is an ongoing prospective collection of placentas in a tertiary center, and our data showed that the placentas with hypertensive disorders represent approximately $12.8 \%$ of the total placentas 
we received with or without decidual vasculopathy, and placentas associated with decidual vasculopathy and other complications without hypertension/preeclampsia $15.4 \%$ (data not shown). It appears that decidual vasculopathy is associated with a series of pregnancy complications including hypertensive disorders, which represent less than one half of the placentas with decidual vasculopathy in our data. These data suggest that decidual vasculopathy in placenta poses additional risks for pregnancy complications other than hypertensive disorders alone, and decidual vasculopathy may or may not directly related to maternal hypertension mechanistically. Consequently, decidual vasculopathy alone is probably not equivalent to maternal vascular malperfusion or underperfusion, and the term "maternal vascular malperfusion/underperfusion" should be a constellation of morphological changes leading to small placentas and vascular ischemia, which seems to fit with early-onset but not late-onset preeclampsia.

The placentas with decidual vascular changes before 20 weeks are not known to be associated with clinical manifestations of preeclampsia. Decidual vascular changes have been seen in placentas up to 24 to 28 weeks delivered for nonpreeclampsia reasons. If the relationship between the persistence of endovascular trophoblasts from early implantation and the decidual vasculopathy at the late gestation are causal, then decidual vasculopathy alone is unlikely to cause maternal hypertension since trophoblastic remodeling of the spiral artery is physiological. It is important to note that endothelial cells in the decidual vessels undergo disappearance at implantation and reappearance at the delivery, and there is an intimate relationship between the invading fetal trophoblasts and the maternal vascular endothelial cells. It is also important to note that the entire placenta is lined by the syncytiotrophoblasts at the intervillous spaces, with direct contact with the maternal circulation, and there is no smooth muscle within these intervillous spaces that can be regulated through the classic local nitric oxide or systemic maternal rennin-angiotensin mechanism. The role of these syncytiotrophoblasts at the intervillous spaces in regulating maternal blood pressure remains largely unclear.

Examination of postpartum uterus and the spiral arteries from the hysterectomy specimens for placenta percreta and postpartum hemorrhage revealed that the spiral arteries within the decidua and inner myometrium largely returned to normal calibers without the apparent presence of endovascular trophoblasts (normal involution, data not shown). However, the term "involution" depicts the rapid reduction of the uterus in size and weight after delivery, with a return to the physiological state of circulation and other functions of the maternal system. Restoration of the uterine spiral artery to the normal state after delivery is only a part of the involution of the maternal systems including the endothelial cell, smooth muscle, and elastic layers of the vessels, in addition to the size and weight of the uterus. "Failure of involution" in the title captures only a fraction of the meaning of involution due to a lack of better term. However, involution may have significant implications for increased incidence of systemic hypertension and cardiovascular disease risks subsequently after preeclampsia.

\section{Natural Killer Cells and Early Pregnancy}

Embryonic implantation occurs at the blastocyst stage with attachment to the endometrial surface and invasion into the endometrium with concurrent differentiation of trophectoderm and inner cell mass. The cytotrophoblasts are differentiated under genetic controls into extravillous or interstitial trophoblasts with potential to invade into the maternal vessels. It is well recognized that NK cells are critical for initiating spiral artery remodeling and that IFN-G is essential for trophoblastic invasion into the vessel walls. ${ }^{20}$ IFN-G is the major cytokine produced in NK cells during pregnancy. In mice model, knockout of IFN-G resulted in the failure of early embryo implantation and loss of pregnancy, only for the first pregnancy (primiparous, $43 \%$ ) but not the second or subsequent pregnancies (multiparous). ${ }^{19}$ IFN-G in mice is peaked at the midgestation (postconception day 10 in a 21-day cycle), and NK cells are the main source of the IFN-G production. In mice model with the absence of NK cells and T cells, there are still detectable levels of IFN-G, indicating that a different cell type other than NK cell or T cells also produces IFN-G during pregnancy. The IFN-G deficient mice also showed decidual arteriole wall thickening with narrowing lumen morphologically similar to the mural artery hypertrophy in human placenta, in addition to decidual necrosis. Knockout mice for IFN-G receptor $\alpha$ were found to have normal implantations and litter sizes, indicating that the function of IFN-G at implantation is not mediated through IFN-G receptor $\alpha$. Mice deficient for both IFN-G and IFN-G receptor $\alpha$ showed increased numbers but hypogranulated NK cells within the uterus during pregnancy.

The function of NK cells in the mouse and the rat model was demonstrated by injecting monoclonal antibody into the abdominal cavity at day 4.5 to day 5 (postconception in a 21-day cycle, around embryonic implantation) or day 9.5 to day 10 (midgestation) of the rats. ${ }^{23,24} \mathrm{NK}$ cells can be effectively reduced in number by injecting specific monoclonal antibody against NK cells. In this model, decidual vasculopathy (fibrinoid medial necrosis and atherosis similar to human decidual vasculopathy) can be found in $67 \%$ rats at term. ${ }^{23,24}$ The effect of NK cells in the midgestation on decidual vasculopathy is difficult to compare with human placenta due to the lack of human decidual vasculopathy data in mid- to late gestation. The fetal weight is decreased significantly at term by reducing the NK cells. Maternal blood pressure and renal functions of the rats were not affected. Reduction of NK cells appeared to reduce expressions of angiogenic factors including vascular endothelial growth factors at both RNA levels and protein levels. $^{23}$

It seems that lack of IFN-G only minimally affects implantation and fetal loss, and lack of NK cells has no effect on the implantation or trophoblastic invasion of the spiral artery in mice or rat model. In the absence of NK cells, the endothelial cells within the spiral artery appear intact in the presence of trophoblasts. ${ }^{23}$ This finding suggests that NK cell function is related to endovascular trophoblasts and endothelial cell interaction, leading to the disappearance and reappearance of endothelial cells in the maternal spiral artery. Examination 
of interaction between the endovascular trophoblasts, CD56 expression, and endothelial cells in the mice or rat model could help answer these questions. NK cells appear important in maintaining the pregnancy in late gestation and fetal growth.

CD56 expression on endovascular trophoblasts, invasion into the vascular lumen, and disappearance of endothelial cells occur simultaneously, and it is possible that CD56 acquisition by the trophoblasts is related to endothelial cell function in early pregnancy. The fact that the trophoblasts replace the endothelial cells at implantation and that the endothelial cells replace trophoblasts at the delivery suggests two separate physiological processes, and abnormalities of both processes can lead to different pathological features. All these changes occur within the maternal spiral artery. It seems possible to measure endothelial cell death in early pregnancy, and trophoblasts cell death later, to gain important information on the implantation and recovery/ preeclampsia.

\section{Human Leukocyte Antigen C and NK Cell Receptors in Preeclampsia}

NK cells express a family of killer Ig-like receptors (KIRs) that structurally belongs to Ig gene family. ${ }^{25-27}$ KIR system is primarily expressed on NK cells and a small subset of T cells. KIR system on the NK cells is divided into activating KIR and inhibitory KIR, and the functions of these KIRs on NK cells are regulated by the receptor ligands. The natural ligands for KIR are class I human leukocyte antigen (HLA) molecules. Unlike NK cells in circulation, the function of uterine NK cells is mainly to produce cytokines and growth factors to regulate implantation and placental development of the fetal trophoblasts. The natural ligand for KIR on the uterine NK cells is predominantly HLA-C, which is expressed on the fetal trophoblasts, and HLA-C expression on the trophoblasts appears to be regulated by both maternal and paternal genes. ${ }^{27,28}$ The complex interaction of KIR and HLA-C in addition to complex KIR gene structures and expressions on NK cells may lead to preeclampsia and other pregnancyrelated complications such as intrauterine growth restriction, recurrent miscarriage, and diabetes. ${ }^{29-31}$ There are many recent reviews on this topic; our interest in KIR system is centered on decidual vasculopathy in preeclampsia and others pregnancy-related complications, ${ }^{25,32}$ and decidual vasculopathy appears to result from the combination of the endovascular fetal trophoblasts and CD56 expression provided by the maternal uterine NK cells. It is plausible that persistence of the endovascular trophoblasts in decidual vasculopathy results from the specific interaction of KIR and HLA-C, leading to fetal trophoblasts, rather than uterine NK cells, to survive longer. It is also worth noting that CD56 itself is an Ig-like glycoprotein expressed on the membrane of NK cells, and whether or not CD56 serves as a receptor for an unknown natural ligand remains an interesting possibility. Direct transfer of CD56 from maternal NK cells to fetal trophoblasts upon invasion into the vessels may have additional significance.

\section{Summary}

Direct transfer of CD56 from the uterine NK cells to fetal trophoblasts upon invasion into the spiral artery in early implantation and subsequent persistence of fetal trophoblasts in decidual vasculopathy in preeclampsia and other pregnancyrelated complications provides interesting insight into the mechanism of pathogenesis of preeclampsia, especially lateonset preeclampsia and other pregnancy-related complications, and offers new directions for research potentially with impact to understanding of the disease.

\section{Conflict of Interest}

None.

\section{References}

1 Lisonkova S, Joseph KS. Incidence of preeclampsia: risk factors and outcomes associated with early- versus late-onset disease. Am J Obstet Gynecol 2013;209(06):544.e1-544.e12

2 Mustafa R, Ahmed S, Gupta A, Venuto RC. A comprehensive review of hypertension in pregnancy. J Pregnancy 2012;2012: 105918

3 Valensise H, Vasapollo B, Gagliardi G, Novelli GP. Early and late preeclampsia: two different maternal hemodynamic states in the latent phase of the disease. Hypertension 2008;52(05):873-880

4 Crispi F, Domínguez C, Llurba E, Martín-Gallán P, Cabero L, Gratacós E. Placental angiogenic growth factors and uterine artery Doppler findings for characterization of different subsets in preeclampsia and in isolated intrauterine growth restriction. Am J Obstet Gynecol 2006;195(01):201-207

5 Benirschke K, Burton GJ, Baergen RN eds. Pathology of the Human Placenta. BerlinSpringer Verlag2012

6 Zhang P, Schmidt M, Cook L. Maternal vasculopathy and histologic diagnosis of preeclampsia: poor correlation of histologic changes and clinical manifestation. Am J Obstet Gynecol 2006;194(04): 1050-1056

7 Hertig A. Vascular pathology in the hypertensive albuminuric toxemias of pregnancy. Clinics 1945;4:602-614

8 Marais W. Human decidual spiral artery studies-part i. Int J Obstet Gynecol 1962;69(02):1-12

9 Marais W. Human decidual spiral arterial studies-part ii. Int J Obstet Gynecol 1962;69(02):213-224

10 Staff AC, Dechend R, Pijnenborg R. Learning from the placenta: acute atherosis and vascular remodeling in preeclampsia-novel aspects for atherosclerosis and future cardiovascular health. Hypertension 2010;56(06):1026-1034

11 Robertson WB, Brosens I, Dixon HG. The pathological response of the vessels of the placental bed to hypertensive pregnancy. J Pathol Bacteriol 1967;93(02):581-592

12 Pijnenborg R, Vercruysse L, Hanssens M, Brosens I. Endovascular trophoblast and preeclampsia: a reassessment. Pregnancy Hypertens 2011;1(01):66-71

13 Brosens I, Robertson WB, Dixon HG. The physiological response of the vessels of the placental bed to normal pregnancy. J Pathol Bacteriol 1967;93(02):569-579

14 Kam EP, Gardner L, Loke YW, King A. The role of trophoblast in the physiological change in decidual spiral arteries. Hum Reprod 1999;14(08):2131-2138

15 Zhang P. CD56 expression of intravascular trophoblasts defines a class of vasculopathy in preeclampsia and other pregnancy complications. https://www.biorxiv.org/content/biorxiv/early/ 2018/04/01/293027.full.pdf2018. Accessed October 16, 2018

16 Waite LL, Atwood AK, Taylor RN. Preeclampsia, an implantation disorder. Rev Endocr Metab Disord 2002;3(02):151-158 
17 Khong TY, Mooney EE, Ariel I, et al. Sampling and definitions of placental lesions: Amsterdam placental workshop group consensus statement. Arch Pathol Lab Med 2016;140(07):698-713

18 Hecht JL, Zsengeller ZK, Spiel M, Karumanchi SA, Rosen S. Revisiting decidual vasculopathy. Placenta 2016;42:37-43

19 Croy BA, Ashkar AA, Minhas K, Greenwood JD. Can murine uterine natural killer cells give insights into the pathogenesis of preeclampsia? J Soc Gynecol Investig 2000;7(01):12-20

20 Ashkar AA, Di Santo JP, Croy BA. Interferon gamma contributes to initiation of uterine vascular modification, decidual integrity, and uterine natural killer cell maturation during normal murine pregnancy. J Exp Med 2000;192(02):259-270

21 Redline RW, Boyd T, Campbell V, et al; Society for Pediatric Pathology, Perinatal Section, Maternal Vascular Perfusion Nosology Committee. Maternal vascular underperfusion: nosology and reproducibility of placental reaction patterns. Pediatr Dev Pathol 2004;7(03):237-249

22 Norwitz ER, Schust DJ, Fisher SJ. Implantation and the survival of early pregnancy. N Engl J Med 2001;345(19):1400-1408

23 Chakraborty D, Rumi MA, Konno T, Soares MJ. Natural killer cells direct hemochorial placentation by regulating hypoxia-inducible factor dependent trophoblast lineage decisions. Proc Natl Acad Sci U S A 2011;108(39):16295-16300

24 Golic M, Haase N, Herse F, et al. Natural killer cell reduction and uteroplacental vasculopathy. Hypertension 2016;68(04):964-973
25 Colucci F. The role of KIR and HLA interactions in pregnancy complications. Immunogenetics 2017;69(8-9):557-565

26 Moffett A, Colucci F. Uterine NK cells: active regulators at the maternal-fetal interface. J Clin Invest 2014;124(05): $1872-1879$

27 Béziat V, Hilton HG, Norman PJ, Traherne JA. Deciphering the killer-cell immunoglobulin-like receptor system at super-resolution for natural killer and T-cell biology. Immunology 2017;150 (03):248-264

28 Moffett A, Chazara O, Colucci F. Maternal allo-recognition of the fetus. Fertil Steril 2017;107(06):1269-1272

29 Hiby SE, Walker JJ, O'shaughnessy KM, et al. Combinations of maternal KIR and fetal HLA-C genes influence the risk of preeclampsia and reproductive success. J Exp Med 2004;200(08): 957-965

30 Hiby SE, Apps R, Sharkey AM, et al. Maternal activating KIRs protect against human reproductive failure mediated by fetal HLA-C2. J Clin Invest 2010;120(11):4102-4110

31 Hiby SE, Regan L, Lo W, Farrell L, Carrington M, Moffett A. Association of maternal killer-cell immunoglobulin-like receptors and parental HLA-C genotypes with recurrent miscarriage. Hum Reprod 2008;23(04):972-976

32 Colucci F, Traherne J. Killer-cell immunoglobulin-like receptors on the cusp of modern immunogenetics. Immunology 2017;152 (04):556-561 